\title{
Some Properties of Generalized Star Semi $\wedge$-Closed Sets in Topological Spaces
}

\author{
M. Matheswaran, S. Rajakumar
}

\begin{abstract}
In this article, we proposed and examined topological characters of $g{ }^{*} \mathrm{~s} \Lambda$-closed, open sets. Its rapport with more generalized closed sets also inspected.
\end{abstract}

Keywords: $g *$ s $\Lambda$-closed sets, $g *$ s $\Lambda$-open sets, $\lambda$ - closed sets, A-closed sets.

\section{INTRODUCTION}

Generalized open sets hits significant aspect in general topology. In 1986, Author [8] advanced Authors [6], [7], [1], [2] work and popularized the perception of $\Lambda$-sets in topological spaces. " $\wedge$-set is a set $\mathrm{A}$ which is equal to its kernel (=saturated set)". Authors [5], [3], [4], [9], [10], [11] deliberated various closed and open sets concept by embroil $\Lambda$-sets and closed sets.

\section{PRELIMINARIES}

In this article $(N, \tau)$, (or simply $N$ ) consistently stands for topological spaces. We recognize some established definitions mandatory for this paper.

\section{Lemma 2.1}

$M \subset N$, succeeding allegations are identical.

1. $M$ is $\lambda$-closed.

2. $M=L \cap \operatorname{cl}(M), L$ is $\Lambda$-set.

3. $M=M^{\Lambda} \cap \operatorname{cl}(M)$.

\section{Lemma 2.2}

$1 . \Lambda$-set is $\lambda$-set.

2.Open, closed are $\lambda$-closed.

\section{Lemma 2.3}

In $T_{1 / 2}$ space every subset of $\mathrm{N}$ is $\lambda$ closed.

\section{Lemma 2.4}

Subset of $T_{1 / 4}$ space is $\lambda$ closed.

Revised Manuscript Received on December 15, 2019.

* Correspondence Author

M. Matheswaran*, Department of Mathematics, Kalasalingam Academy of Research and Education, Krishnankoil, Tamilnadu, India. Email:mathes.maths@gmail.com

S. Rajakumar, Department of Mathematics, Kalasalingam Academy of Email: srkumar277@gmail.com Research and Education, Krishnankoil, Tamilnadu, India.

\section{PROPERTIES OF $g^{*} \boldsymbol{S} \Lambda$ - CLOSED SETS}

Definition 3.1 $M \subseteq N$ is $g^{*} s \Lambda$-closed if $c l_{\lambda}(M) \subset Q$ whenever $M \subset Q, Q$ is $g s$-open in $N$.

Theorem 3.2 $\lambda$-closed $\Rightarrow g^{*} s \Lambda$-closed.

Proof. Consider $M \subset Q, Q$ is $g s$-open.

$\therefore M$ is $\lambda$-closed. Hence $c l_{\lambda}(M)=M \subset Q$.

$\Rightarrow M$ is $g^{*} \Lambda \Lambda$-closed.

Remark 3.3 $g^{*} s \Lambda$-closed $\neq \lambda$-closed by subsequent example.

Example 3.4 Take $N=\{\gamma, \eta, \lambda, \sigma\}$ with $\tau=\{N, \phi,\{\gamma\},\{\eta$, $\lambda\},\{\gamma, \eta, \lambda\},\{\eta, \lambda, \sigma\}\}$. Here $\{\eta, \sigma\}$ is not $\lambda$ closed but $g^{*} s \Lambda$-closed.

Theorem 3.5 Closed $\Rightarrow g^{*} s \Lambda$ closed.

Proof. Proof pursued from "lemma 2.2 and theorem 3.2".

Remark 3.6 $g^{*} s \Lambda$-closed is not closed by ensuing example.

Example 3.7 Let $N=\{\psi, \mu, \eta, \gamma\}$ and $\tau=\{N, \phi,\{\psi\},\{\mu, \eta\}$, $\{\psi, \mu, \eta\},\{\mu, \eta, \gamma\}\}$. Here $\{\mu, \gamma\}$ is $g^{*} s \Lambda$ closed but not closed.

Theorem 3.8 Open $\Rightarrow g^{*} s \Lambda$-closed.

Proof. Proof conspicuous by definitions.

Theorem $3.9 g s$-open $\Rightarrow \lambda$-closed if it is $g^{*} s \Lambda$-closed.

Proof. Consider $M$ be $g^{*} s \Lambda$-closed with $g s$-open.

$\because M \subset M, c l_{\lambda}(M) \subset M$.

$\Rightarrow M$ is $\lambda$ closed.

Theorem 3.10 $g^{*} s \Lambda$-closed $\Rightarrow g \Lambda$-closed.

Proof. Certify $M$ be $g^{*} s \Lambda$-closed, $M \subset Q, Q$ is open. Open $\Rightarrow g s$-open, also $M$ is $g^{*} s \Lambda$-closed.

$\Rightarrow c l_{\lambda}(M) \subset \mathrm{Q}$

$\Rightarrow M$ is $g \Lambda$-closed.

Remark 3.11 $g \Lambda$-closed $\neq g^{*} s \Lambda$-closed by coming example.

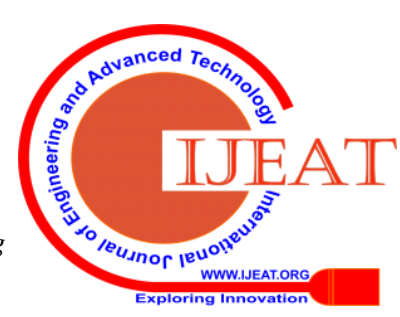


Example 3.12 Take $N=\{v, \sigma, \rho\}$ and $\tau=\{N, \varphi,\{v\},\{v$, $\rho\}$. Then $\{v, \sigma\}$ is not $g^{*} s \Lambda$ - closed but $g \Lambda$-closed.

Remark 3.13 Generalized closed and $g^{*} s \Lambda$-closed are self-reliant.

Remark 3.14 $g^{*} s \Lambda$-closed, $\omega$-closed are autonomous.

Theorem 3.15 $\omega$-closed $\Rightarrow g^{*} S \Lambda$-closed, if $g s$ open is semi open.

Proof. Consider $M \subset Q, Q$ is $g s$-open.

$\because M$ is $\omega$-closed.

$\Rightarrow \operatorname{cl}(M) \subset Q$. But $C l_{\lambda}(M) \subset C l(M) \subset Q$.

$\Rightarrow M$ is $g^{*} s \Lambda$-closed.

Remark 3.16 $g^{*} s \Lambda$-closed, $\Lambda g$-closed are nonpartisan.

Remark 3.17 $g^{*} s \Lambda$-closed, $g s$-closed are self-reliant.

Remark 3.18 $g^{*} s \Lambda$-closed, $g p$-closed are autonomous.

Remark 3.19 $M, L$ are $g^{*} s \Lambda$-closed but $M \cap L$ is not $g^{*} s \Lambda$-closed.

Remark 3.20 $M \cup L$ is not $g^{*} s \Lambda$-closed even though $M, L$ are $g^{*} s \Lambda$-closed.

\section{APPLICATIONS OF $g^{*} s \Lambda$ - CLOSED SETS}

Theorem 4.1 $M$ is $g^{*} s \Lambda$-closed $\Rightarrow c l_{\lambda}(M) \backslash M$ contains empty closed set.

Proof. Consider $M$ be $g^{*} \Lambda \Lambda$-closed.

Suppose $\phi \neq P \subset \operatorname{cl}_{\lambda}(M) \backslash M$.

$\Rightarrow M \subseteq P^{\mathrm{c}}, P^{\mathrm{c}}$ is open.

$\because M$ is $g^{*} s \Lambda$-closed, open $\Rightarrow g s$-open, $c l_{\lambda}(M) \subseteq P^{\mathrm{c}}$.

$\therefore P \subseteq N \backslash c l_{\lambda}(M)$, also $P \subseteq c l_{\lambda}(M)$.

$\Rightarrow P \subseteq\left[N \backslash c l_{\lambda}(M)\right] \cap c l_{\lambda}(M)=\phi$.

$\Rightarrow c l_{\lambda}(M) \backslash M$ contains empty closed set.

Remark 4.2 Reverse part of theorem 4.1 fails by the coming example.

Example 4.3 Assume $N=\{\eta, \theta, \psi, \chi\}$ with $\tau=\{N$, $\phi,\{\eta\},\{\theta\},\{\eta, \theta\},\{\theta, \psi, \chi\}\}$. If $M=\{\eta, \chi\}, \operatorname{cl}_{\lambda}(M)=\{\eta, \psi$, $\chi\}, c l_{\lambda}(M) \backslash M=\{\psi\} \supset \quad$ closed set $\neq \phi$ however $M$ is $g^{*} s \Lambda$-closed.

Theorem 4.4 $M$ is $g^{*} \Lambda \Lambda$-closed $\Rightarrow c l_{\lambda}(M) \backslash M$ contains empty gs-closed set.

Proof. Take $\mathrm{M}$ be $\mathrm{g}^{*} \mathrm{~s} \Lambda$-closed.

Suppose $P$ is a $g s$-closed $\subset c l_{\lambda}(M) M$.

$\Rightarrow M \subseteq P^{\mathrm{c}}$, where $P^{\mathrm{c}}$ is $g s$-open.

Since $M$ is $g^{*} S \Lambda$-closed $c l_{\lambda}(M) \backslash P^{\mathrm{c}}$.

$\Rightarrow P \subseteq \mathrm{N} \backslash c l_{\lambda}(M)$, also $P \subseteq c l_{\lambda}(M)$.
$\Rightarrow P \subseteq\left[N \backslash c l_{\lambda}(M)\right] \cap \quad c l_{\lambda}(M)=\phi$.

$\therefore \mathrm{cl}_{\lambda}(M) \backslash M$ contains empty $g s$-closed set.

Theorem 4.5 Subset of $T_{1 / 2}$ space is $\mathrm{g}^{*} \Lambda \Lambda$-closed.

Proof. Proof evident from lemma 2.3 and theorem 3.2

Theorem 4.6 Subset of $T_{1 / 4}$ space is $g^{*} S \Lambda$ closed.

Proof. Proof results from "lemma 2.4 and theorem 3.2"

Theorem 4.7 In $T_{1}$ space $\Lambda g$-closed is $g^{*} s \Lambda$-closed.

Proof. In $T_{1}$ space $\Lambda g$-closed $\Rightarrow$ closed.

Closed $\Rightarrow g^{*} s \Lambda$-closed "by theorem 3.5 "

$\Rightarrow$ In $T_{1}$ space $\Lambda g$-closed is $g^{*} \Lambda \Lambda$-closed.

Theorem 4.8 Certify $M$ be $g^{*} s \Lambda$-closed. Then $M$ is $\lambda$-closed if and only if $c l_{\lambda}(M) \backslash M$ is closed.

Proof.

Necessity: Consider $M$ be $g^{*} s \Lambda$ closed, $\lambda$ closed.

$M$ is $\lambda$ closed $\Rightarrow c l_{\lambda}(M)=M$.

$\Rightarrow c l_{\lambda}(M) M=\phi$ is closed.

Sufficiency: Take $M$ is $g^{*} S \Lambda$-closed and $c l_{\lambda}(M) \backslash M$ is closed. $\Rightarrow c l_{\lambda}(M) M$ contains empty closed "by theorem 4.1 "

$\Rightarrow c l_{\lambda}(M) \backslash M=\phi$.

$\Rightarrow C l_{\lambda}(M)=M$. Therefore, $M$ is $\lambda$-closed.

Theorem 4.9 If $g^{*} S \Lambda$-closed is $\lambda$-closed then $\{x\}$ is $g s$-closed or $\lambda$-open.

Proof. Assume $\{x\}$ is not $g s$-closed.

$\Rightarrow N \backslash\{x\}$ is not $g s$-open.

$\Rightarrow N$ is the only $g s$-open set $\supset N \backslash\{x\}$.

Evidently $\operatorname{cl}_{\lambda}(N \backslash\{x\}) \subseteq N$.

$\therefore N \backslash\{x\}$ is $g^{*} s \Lambda$-closed.

$\Rightarrow\{x\}$ is $\lambda$-open.

Theorem 4.10 Consider $M$ be $g s$-open and $g^{*} s \Lambda$-closed. $P$ is $\lambda$-closed then $M \cap P$ is $g^{*} s \Lambda$-closed.

Proof. $M$ is both $g s$-open and $g^{*} s \Lambda$-closed $\Rightarrow M$ is $\lambda$-closed "by theorem 3.9 ".

$\Rightarrow M \cap P$ is $\lambda$-closed as the intersection of $\lambda$-closed sets is $\lambda$-closed.

$\Rightarrow M \cap P$ is $g^{*} s \Lambda$-closed "by theorem 3.2".

Theorem 4.11 $M$ is $g^{*} s \Lambda$-closed $\Rightarrow \operatorname{gscl}(\{x\}) \cap M \neq \phi$, $\forall x \in c l_{\lambda}(M)$.

Published By:

Blue Eyes Intelligence Engineering 
Proof. Assume $M$ be $g^{*} s \Lambda$-closed with $\operatorname{gscl}(\{x\}) \cap M=\varnothing$ $\exists x \in c l_{\lambda}(M)$.

$\Rightarrow N \backslash \operatorname{gscl}(\{x\})$ is a $g s$ open $\supset M$.

Also, $x \in \operatorname{cl}_{\lambda}(M)$ and $x \notin N \backslash \operatorname{gscl}(\{x\})$.

$\Rightarrow x \in\left(\operatorname{cl}_{\lambda}(M)\right) \notin N \backslash \operatorname{gscl}(\{x\})$.

$\Rightarrow \operatorname{cl}_{\lambda}(M) \not \subset X \backslash \operatorname{gscl}(\{x\})$.

$\Rightarrow \Leftarrow M$ is a $g^{*} s \Lambda$ closed.

$\Rightarrow \operatorname{gscl}(\{x\}) \cap \mathrm{M} \neq \phi, \forall x \in C l_{\lambda}(M)$

Theorem 4.12 $M$ be $g s$-open. The ensuing's are identical

1. $\lambda$-closed.

2. $g^{*} s \Lambda$-closed.

Proof.

$(1) \Rightarrow(2)$

Assume $M \subset N$ with $M \subseteq Q, Q$ is $g s$-open.

$\Rightarrow \operatorname{cl}_{\lambda}(M) \subseteq c l_{\lambda}(Q)$. Here $Q$ is $\lambda$-closed.

$\Rightarrow c l_{\lambda}(M) \subseteq c l_{\lambda}(Q)=Q$.

$\Rightarrow M$ is $g^{*} s \Lambda$-closed.

(2) $\Rightarrow(1)$

Consider $M$ be $g s$-open with $M$ is $g^{*} s \Lambda$-closed.

$\Rightarrow c l_{\lambda}(M) \subseteq M$.

Theorem 4.13 In door space subset is $g^{*} s \Lambda$-closed.

Proof. Certify $M \subseteq N$.

Since in door space, subset is one among the choices, open or closed.

$\Rightarrow$ Subset is $\lambda$-closed.

$\Rightarrow$ Subset is $g^{*} s \Lambda$ closed "by theorem 3.2".

Theorem 4.14 In partition space, $g^{*} s \Lambda$-closed $\Rightarrow g s$-closed.

Proof. Take $M$ abide $g^{*} s \Lambda$ closed with $M \subseteq Q, Q$ is open.

As open is $g s$-open

$\Rightarrow Q$ is $g s$-open.

But $M$ is $g^{*} s \Lambda$-closed.

$\Rightarrow \operatorname{cl}_{\lambda}(M) \subseteq Q$.

In partition space, closed set is open.

$\therefore \operatorname{cl}(A)=\operatorname{cl}_{\lambda}(M) \subseteq Q$.

$\Rightarrow M$ is $g s$-closed.

\section{V.CHARACTERISTICS OF $g^{*} \boldsymbol{\Lambda} \Lambda$ - OPEN SETS}

Definition 5.1 $M \subseteq N$ is $g^{*} S \Lambda$ open if $M^{\mathrm{c}}$ is a $g^{*} S \Lambda$ closed.

Theorem $5.2 \lambda$ open $\Rightarrow g^{*} s \Lambda$-open.

Proof. Assume $M$ be a $\lambda$ open.

$\Rightarrow N \backslash M$ is $\lambda$-closed.

$\Rightarrow N \backslash M$ is $g^{*} s \Lambda$-closed "by theorem 3.2"”.

$\Rightarrow M$ is $g^{*} S \Lambda$-open.
$\Rightarrow M$ is $\lambda$-closed.

Theorem 5.3 Closed $\Rightarrow g^{*} s \Lambda$ open.

Proof. Consider $M$ be a closed.

$\Rightarrow N \backslash M$ is open.

$\Rightarrow N \backslash M$ is $\lambda$ closed "by lemma 2.2 ".

$\Rightarrow M$ is $\lambda$ open.

$\Rightarrow M$ is $g^{*} s \Lambda$ open "by theorem 5.1".

Theorem 5.4 Open entail $g^{*} s \Lambda$ open.

Proof. Assume $M$ hold open.

$\Rightarrow N \backslash M$ closed.

$\Rightarrow N \backslash M$ is $\lambda$-closed "by lemma 2.2 ".

$\Rightarrow N \backslash M$ is $g^{*} s \Lambda$ closed "by theorem 3.2".

$\Rightarrow M$ is $g^{*} S \Lambda$ open.

\section{ACKNOWLEDGMENT}

The Authors indebted to the referees for their beneficial ideas and conclusions which will help to advance the paper.

\section{REFERENCES}

1. Arokiarani I, Balachandran K, Dontchev J. Some Characterization of $g p$-irresolute and $g p$-continuous maps between topological spaces.Mem Fac Sci Kochi Univ Ser A (Math)1999; 20:93-104

2. S.P.Arya and T. M. Nour, Characterization of S-normal spaces, Indian J.Pure Appl. Math, 21(1990), 717-719

3. M. Caldas, S. Jafari and T. Noiri, On $\Lambda$-generalized closed sets in topological spaces, Acta Math. Hungar, 118(4) (2008),337-343.

4. M. Caldas, S.Jafari and T.Navalagi, More on $\lambda$-closed sets in topological spaces, Revista colombia de Mathematics, Vol 41(2007)2,355-369.

5. Francisco G Arenas, Julian Dontchev and Maxmillan Ganster, On- $\lambda$ closed sets and the dual of generalized continuity, Question answers GEN.Topology 15(1997)3-13.

6. N. Levine, Semi-open sets and semi continuity in topological spaces, Amer. Math. Monthly, 70(1963), 36-41.

7. N. Levine, Generalized closed sets in topology Rend. Circ. Mat. Palermo, 19(1970), 89-96

8. H. Maki, Generalized $\Lambda$-sets and the associated closure operator, The special issue in Commemoration of Prof. Kaszusada IKEDS Retirement, 1 oct (1986), 139-146.

9. M. Sheik John, On $w$-closed sets in Topology, Acta Ciencia Indica 4(2000)389-392.

10. M. K. R. S. Veerakumar, $g *$-Closed sets in topological spaces, Mem Fac, Sci. Kochi Univ. (Math), 21(2000), 1-19

11. M. K. R. S. Veerakumar, On $g$-Closed sets in topological spaces, Bull Alahabad. Soc18(2003)99-112.

\section{AUTHORS PROFILE}

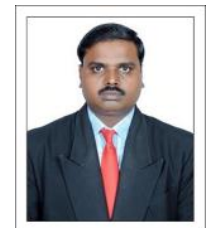

M. Matheswaran is currently working as an Assistant Professor in the Department of Mathematics (School of Advanced Sciences), Kalasalingam Academy of Research and Education (Deemed to be University), Anand Nagar, Krishnankoil, Tamilnadu - 626126. His areas of interest include Topology, Probability and Queuing Theory, Differential Equations, Operations Research, and Discrete Mathematical Structures. He obtained his M.Sc. Degree at Anna University,

Published By:

Blue Eyes Intelligence Engineering 
Some Properties of Generalized Star Semi ^-Closed Sets in Topological Spaces

Chennai also M.Phil. Degree at Bharathidasan University, Tiruchirappalli. He has 14 years of Teaching Experience. He has delivered invited talks and also organized many academic and non-academic events. $\mathrm{He}$ is a member of Mathematical Societies and International Association of Engineers.

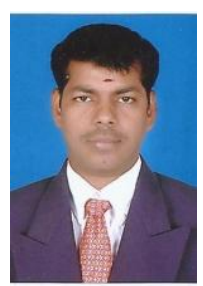

Dr. S. Rajakumar is currently working as an Assistant Professor in the Department of Mathematics (School of Advanced Sciences) in Kalasalingam Academy of Research and Education (Deemed to be University), Krishnankoil-626126. He obtained his Ph.D., degree in Manonmaniam Sundaranar University, Tirunelveli. His area of research interest is Topology and Bitopological spaces. He has $17+$ years of teaching experience. He has a few publications in standard journals. 